\title{
Nonlinear Schrödinger solitons oscillate under a constant external force
}

\author{
Franz G. Mertens \\ Physikalisches Institut, Universität Bayreuth, 95440 Bayreuth, Germany \\ Niurka R. Quintero \\ IMUS and Departamento de Física Aplicada 1, E.P.S Universidad de Sevilla, Virgen de Africa 7, 41011 Sevilla, Spain
}

A. R. Bishop

Los Alamos National Laboratory, Los Alamos, New Mexico 87545, USA

(Received 5 October 2012; revised manuscript received 12 February 2013; published 27 March 2013)

\begin{abstract}
We investigate the dynamics of solitons of the cubic nonlinear Schrödinger equation with an external timeindependent force of the form $f(x)=r \exp (-i K x)$. Here the solitons travel with an oscillating velocity and all other characteristics of the solitons (amplitude, width, momentum, and phase) also oscillate. This behavior was predicted by a collective variable theory and confirmed by simulations. However, the reason for these oscillations remains unclear. Moreover, the spectrum of the oscillations exhibits a second strong peak, in addition to the intrinsic soliton peak. We show that the additional frequency belongs to a certain extended linear mode (which we refer to as a phonon for short) close to the lower band edge of the phonon continuum. Initially the soliton is at rest. When it starts to move it is deformed, begins to oscillate, and excites the above phonon mode such that the total momentum in a certain moving frame is conserved. In this frame the phonon does not move. However, not only does the soliton move in the homogeneous, time-periodic field of the phonon, but it also oscillates.
\end{abstract}

DOI: 10.1103/PhysRevE.87.032917

PACS number(s): $05.45 . \mathrm{Yv}$

\section{INTRODUCTION}

The nonlinear Schrödinger equation (NLSE) has numerous applications covering practically all fields of physics, which are listed and discussed in several review articles [1-3]. For many applications it is important to study the driven NLSE. Parametric drivings were considered in Refs. [1,2,4-8]. Nonparametric (external) drivings were studied using timedependent forces, e.g., $f(t)=r \exp (i \omega t)$ [9-11], or a spaceand time-dependent force in the form of a plane wave $f(x, t)=$ $r \exp [i(k x-\omega t)][12,13]$. The externally driven NLSE arises in many physical situations such as charge density waves [9], long Josephson junctions [14], optical fibers [12,15], and plasmas driven by rf fields [16].

Recently, a force of the form $f(x, t)=r \exp [-i K(t) x]$ was considered, where $K(t)$ was a harmonic or biharmonic function $[17,18]$. Such forces were first used in the discrete form $f_{n}(t)=r \exp [-i n \phi(t)]$, where $n$ denotes the $n$th resonator in an array of coupled nonlinear optical waveguides, in which discrete cavity solitons can be excited [19]. The array can be modeled by a discrete NLSE, where $\phi$ is the incident angle of a laser pump light. Both harmonic and biharmonic functions $\phi(t)$ were considered [20].

Interestingly, the special case of a time-independent force $f(x)=r \exp (-i K x)$ yields time-depending oscillating soliton solutions, i.e., the amplitude, width, velocity, momentum, and phase of the soliton all oscillate [17]. We study the following forced NLSE

$$
i \psi_{t}+\psi_{x x}+g|\psi|^{2} \psi+\delta \psi=r e^{-i K x},
$$

where $g, \delta$, and $K$ are constants. In the above-mentioned discrete NLSE, which models cavity solitons, $\delta<0$ is a cavity detuning parameter [19]. In our simulations [17], we obtained stable solitons only for $\delta<0$.
Our collective coordinate (CC) theory for Eq. (1) predicts soliton oscillations for nearly all initial conditions (ICs). This has been confirmed by our simulations for very long integration times $[17,18]$. For IC, we take the exact one-soliton solution of the unperturbed NLS equation, i.e., Eq. (1) with $r=0$. If the initial condition is a stable stationary solution of the $\mathrm{CC}$ equations, the simulations exhibit very small harmonic oscillations. If the IC is close to a stable stationary solution, the $\mathrm{CC}$ equations predict nearly harmonic oscillations, which is confirmed by our simulations. If the IC is not close to a stationary solution, there are two possibilities: either anharmonic oscillations are predicted and confirmed (see Fig. 6 of Ref. [17]), or very anharmonic oscillations are predicted, but the soliton becomes unstable after some time (Fig. 4 of Ref. [17]).

These instabilities can be predicted by an empirical criterion, which is based on the CC theory [18]. The soliton becomes unstable if the "stability curve" $p(v)$, where $p(t)$ and $v(t)$ are the normalized momentum and the velocity of the soliton, has a section with a negative slope. This criterion has a wide validity. It also holds for the NLSE with the nonlinearity [21] $g\left(\psi^{*} \psi\right)^{\kappa} \psi$ with $\kappa=1 / 2$, and it also holds if $K$ is not constant but a harmonic or biharmonic function of time [18]; here $\kappa=1$.

Two questions have not been clarified in the above work [17,18,21]: (1) Why do the solitons oscillate, although the driving force is time independent? (2) In the simulations, the spectrum of the oscillations in most cases exhibits two strong peaks, but only one of them is located near the frequency $\omega_{s}^{c c}$ which is predicted by the CC theory. What is the origin and the role of the second peak?

The spatial periodicity of the driving force $r e^{-i K x}$ is not the reason for the oscillations. The period $2 \pi /|K|$ is not reflected in the soliton dynamics, because Eq. (1) can be reduced to an 
autonomous equation in a moving frame [17]. Assuming

$$
\psi(x, t)=e^{-i K x} u(y, t), \quad y=x+2 K t,
$$

then Eq. (1) becomes

$$
i u_{t}+u_{y y}+g|u|^{2} u-\left(K^{2}-\delta\right) u=r .
$$

Indeed, simulations for Eq. (3) yield the same soliton oscillations as Eq. (1). The only difference is that the soliton velocity oscillates around a different mean value. The value of $K^{2}-\delta$ must be positive, otherwise the homogeneous solution $u(y, t) \equiv u_{0}$ would be unstable [11]

Equation (3) has a history of applications, in particular, in the physics of optical cavities. Originally, it was introduced as the Lugiato-Levefer model [22] of a diffractive cavity driven by a plane-wave stationary beam. Later it was employed to describe a synchronously pumped ring laser with a nonlinear dispersive fiber [23,24]. More recently, the same equation was shown to govern the envelopes of short baroclinic Rossby waves in a two-layer model of the atmosphere or of the ocean [25].

Equation (3) has two exact static solutions [11,21,26]:

$$
u_{ \pm}(y, t)=a+\frac{b}{c \pm \cosh (\beta y)},
$$

where $a, b, c$, and $\beta$ are functions of the parameters $g, r$, and $K^{2}-\delta=K^{\prime 2}$

A numerical linear stability analysis [11] shows that $u_{+}(y, t)$ is always unstable. The solution $u_{-}(y, t)$ is stable below a critical value $r_{c}$ of the driving strength. For $0<r<r_{c}$, there are two eigenfrequencies in the gap between the continuum of linear excitations (henceforth referred to as phonons) and the ground state. For small $r$, the lower frequency is proportional to $\sqrt{r}$, the higher frequency mode is detached from the continuum, and the distance from the continuum increases linearly with $r$. However, the above static solutions of the autonomous equation (and small oscillations around them) cannot answer our question, because our oscillating solitons are moving also in the moving frame, i.e., the soliton velocity $v_{s}$ generally differs from the velocity of the moving frame $v_{f}=-2 K$.

In a recent paper [27], traveling solitons of the autonomous equation were studied numerically by path-following them in the parameter space. However, since these traveling solitons do not exhibit oscillations, neither can they explain the oscillating solitons which were predicted by our CC theory [17] and confirmed by our simulations.

In this paper we essentially employ the following two ideas: In most of our simulations the soliton is initially at rest. The external driving force has two effects:

(1) The soliton is accelerated and starts to move. Due to the acceleration the soliton is deformed and starts to oscillate. This means that an eigenmode with frequency $\omega_{s}$ is excited.

(2) Initially the momentum of the system is zero, because the soliton is at rest and there are no other excitations. When the soliton starts to move, it acquires a momentum $P_{s}$ and a plane wave (a linear excitation) can be excited such that the total momentum in the moving frame is conserved. The soliton then can move in the inhomogeneous, time-periodic field of the plane wave. We note that much earlier there was a very similar problem studied with driven nonplanar vortices on a circular easy-plane Heisenberg ferromagnet. There the vortices perform oscillations (with two frequencies) around a mean trajectory, which is a circle. The oscillations occur due to the interaction of the vortex with two spin-wave modes, which are excited at the beginning of the simulation when the vortex stars to move, in order to conserve the total energy and total angular momentum [28]. A circular magnet is spatially inhomogeneous due to the Coulomb force between the vortex and its image vortex.

Our paper is organized as follows: In Sec. II we deal with the momentum conservation and calculate the norm and the momentum of the soliton. The spectrum, momentum, and norm of the phonon modes are calculated in Sec. III. It turns out that a certain phonon mode close to the lower band edge is the only relevant mode for the soliton oscillations. In the moving frame, this mode is a homogeneous oscillation, i.e., this phonon does not move in the moving frame.

The theoretical results are confirmed by simulations [direct numerical solutions of the NLSE (1) and (3)] in Sec. IV. A discrete Fourier transform (DFT) of the simulation data yields the spectrum of the soliton oscillations as a function of the driving strength $r$. The spectrum consists of a soliton eigenmode and the above phonon near the band edge.

\section{MOMENTUM CONSERVATION}

As the force $f(x)=r \exp (-i K x)$ in Eq. (1) does not depend on time, the energy is conserved [17]. The expression for the energy is given in Sec. IV. The norm and the momentum of the NLS soliton are, respectively,

$$
M=\int d x \psi^{*} \psi
$$

and

$$
P=\frac{i}{2} \int d x\left(\psi \psi_{x}^{*}-\psi^{*} \psi_{x}\right) .
$$

In Ref. [21] it was proven that

$$
P(t)+K M(t)=P_{v}=\text { const, }
$$

where

$$
P_{v}=\frac{i}{2} \int d x\left(u u_{y}^{*}-u^{*} u_{y}\right)
$$

is the momentum in the frame moving with $v_{f}=-2 K$. The conservation law (7) holds under the condition that the boundary terms $F(y \rightarrow+\infty, t)$ and $F(y \rightarrow-\infty, t)$ are the same (or zero). Here, $F=-j+r u+r u^{*}$, where $j(y, t)$ is the current density of the unperturbed NLS Eq. (3) in the moving frame [21].

In our simulations we use a finite system of length $2 L$ and periodic boundary conditions. We now assume that in the presence of a moving soliton there is always a plane wave phonon (short for a linear excitation) and that

$$
P=P_{s}+P_{\mathrm{ph}}, \quad M=M_{s}+M_{\mathrm{ph}} .
$$

In the following we calculate separately the soliton and phonon contributions. 


\section{A. Soliton momentum and norm in the $\mathrm{CC}$ theory}

We use the CC ansatz of Ref. [21] for the NLS equation with arbitrary nonlinearity $\kappa$, but we set $\kappa=1$, which corresponds to our NLS equation in Eq. (1):

$$
\psi(x, t)=\beta \operatorname{sech}[\beta(x-q)] e^{i[p(x-q)+\phi]},
$$

with amplitude $\beta(t)$ equal to the inverse of the width, position $q(t)$, normalized momentum $p(t)$, and phase $\phi(t)$. Note that $\psi(x, t)$ is an exact solution of the unperturbed NLS equation. It turns out that two of the four ordinary differential equations for the collective variables are not independent [21], which allows us to carry out one integration. This yields [21]

$$
\beta(t)[p(t)+K]=\text { const. }
$$

Inserting the ansatz (10) into $M$ and $P$ given by (5) and (6), respectively, we obtain the soliton norm and momentum in the $\mathrm{CC}$ theory,

$$
M_{s}^{c c}(t)=2 \beta(t), \quad P_{s}^{c c}(t)=2 \beta(t) p(t) .
$$

Using (11), we see that the conservation law (7) is fulfilled by the $\mathrm{CC}$ theory

$$
P_{s}^{c c}(t)+K M_{s}^{c c}(t)=\text { const, }
$$

where the constant is determined by the initial conditions

$$
P_{v}^{c c}=2 \beta_{0}\left(p_{0}+K\right),
$$

and equals the soliton momentum in the moving frame.

\section{SPECTRUM OF THE LINEAR EXCITATIONS (PHONONS)}

The general solution of the linearized NLSE (Eq. (73) of [17]) reads

$$
\psi_{\mathrm{ph}}(x, t)=c e^{i\left(k x-\omega_{k} t\right)}-\frac{r}{\omega_{K}} e^{-i K x},
$$

with $\omega_{k}=-\delta+k^{2}, \omega_{K}=-\delta+K^{2}$, and arbitrary, but small $c$. The lower edge of the phonon continuum is at $\omega_{0}=-\delta$, and therefore the spectrum contains a gap.

\section{A. Phonons in the presence of a soliton}

For the following, we need to know these phonons only far away from the soliton. We assume that there is a phase shift $\theta$ due to the interaction with the soliton, i.e., at the far left we obtain

$$
\psi_{\mathrm{ph}}(x, t)=c e^{i\left(k x-\omega_{k} t+\theta\right)}-\frac{r}{\omega_{K}} e^{-i K x},
$$

and at the far right the phonons are represented by Eq. (15).

\section{B. Phonon momentum in the presence of a soliton}

Equations (15) and (16) are inserted into $P$ and $M$, defined by Eqs. (6) and (5), respectively. We must distinguish two cases: $k \neq-K$ and $k=-K$. In the former case the results are complicated, but one can see in any case that

$$
P_{\mathrm{ph}}(t)+K M_{\mathrm{ph}}(t) \neq \text { const. }
$$

In the case $k=-K$, the calculations are much simpler, because the $x$-dependent parts in the integrands of $P$ and $M$ drop out, and moreover $\theta$ drops out. Indeed,

$$
\psi_{\mathrm{ph}}(x, t)=\left(c e^{-i \omega_{K} t}-\frac{r}{\omega_{K}}\right) e^{-i K x}=\phi_{\mathrm{ph}}(t) e^{-i K x},
$$

with $\omega_{-K}=\omega_{K}=K^{2}-\delta$. First consider $M_{\mathrm{ph}}(t)$ :

$$
\begin{aligned}
M_{\mathrm{ph}}(t) & =\int_{-L}^{+L} d x \psi_{\mathrm{ph}} \psi_{\mathrm{ph}}^{*}=\left.\phi_{\mathrm{ph}}(t) \phi_{\mathrm{ph}}^{*}(t) x\right|_{-L} ^{+L}, \\
& =\left(|c|^{2}+\frac{r^{2}}{\omega_{K}^{2}}\right) 2 L-2 \operatorname{Re}\left(\frac{c r}{\omega_{K}} e^{-i \omega_{K} t}\right) 2 L .
\end{aligned}
$$

In the same way

$$
\begin{aligned}
P_{\mathrm{ph}}(t) & =\int_{-L}^{+L} d x \operatorname{Im}\left(\frac{\partial \psi_{\mathrm{ph}}}{\partial x} \psi_{\mathrm{ph}}^{*}\right) \\
& =-K\left(|c|^{2}+\frac{r^{2}}{\omega_{K}^{2}}\right) 2 L+2 K \frac{r}{\omega_{K}} \operatorname{Re}\left(c e^{-i \omega_{K} t}\right) 2 L .
\end{aligned}
$$

Thus

$$
P_{\mathrm{ph}}(t)+K M_{\mathrm{ph}}(t)=0,
$$

for phonons with $k=-K, \omega_{K}=K^{2}-\delta$. The right-hand side of (23) is the momentum in the moving frame. It is zero, because the phonon does not travel in this frame: the group velocity in the laboratory frame is $v_{k}=\frac{d \omega_{k}}{d k}=2 k$. For the mode with $k=-K$ this velocity is $v_{\mathrm{ph}}=-2 K$. The velocity of the moving frame is $v_{f}=-2 K$. The phonon velocity in this frame is

$$
\tilde{v}_{\mathrm{ph}}=v_{\mathrm{ph}}-v_{f}=0 .
$$

Now consider an oscillating soliton plus a phonon with $k=$ $-K$. We assume that the momenta and the norms of the soliton and the phonons are additive:

$$
P=P_{s}+P_{\mathrm{ph}}, \quad M=M_{s}+M_{\mathrm{ph}} .
$$

By inserting Eq. (25) into the conservation law Eq. (7) and using Eq. (23), we obtain

$$
P_{s}+K M_{s}=P_{v} .
$$

Thus in the moving frame the (total) momentum is equal to the soliton momentum, and there is no contribution from the phonon. The phonon is simply a homogeneous oscillation, i.e., without $x$ dependence:

$$
\phi_{\mathrm{ph}}(t)=c e^{-i \omega_{K} t}-\frac{r}{\omega_{K}} .
$$

This will be confirmed in Sec. IV by our simulations for the NLS Eq. (3) in the moving frame. This means that the above additivity assumption is justified $a$ posteriori.

In the laboratory frame the phonon is

$$
\psi_{\mathrm{ph}}(x, t)=\phi_{\mathrm{ph}}(t) e^{-i K x},
$$

and moves with the velocity $v_{\mathrm{ph}}=-2 K$.

\section{SIMULATIONS VS THEORY}

In this section, we test our results by simulations, i.e., by numerically solving the NLSE [(1) and (3)]. We have used 
a fourth-order Runge-Kutta method and periodic boundary conditions. The parameters related with the discretization of the system are $\Delta x=0.02, \Delta t=10^{-4}$ [such that $\Delta t<$ $\left.(1 / 2)(\Delta x)^{2}\right]$.

In order to compute the soliton position $q(t)$, we fix the time and determine the value of $x$ for which the density of the norm is a maximum, i.e., $\max \left(|\psi|^{2}\right)$. Hence, we compute $\beta=\sqrt{\max \left(|\psi|^{2}\right)}$. We have also computed the momentum by using (6) and the energy $E(t)$,

$$
E=\int_{-L}^{+L} d x\left(\left|\psi_{x}\right|^{2}-\frac{g}{2}|\psi|^{4}-\delta|\psi|^{2}+f \psi^{*}+f^{*} \psi\right),
$$

which is a conserved quantity [1]. We have verified by decreasing $\Delta x$ and $\Delta t$ that very small oscillations of the energy in the simulations are due to numerical errors.

As initial conditions, we take Eq. (10) with $\beta(0)=$ $\beta_{0}, q(0)=q_{0}, p(0)=p_{0}$, and $\phi(0)=\phi_{0}$. Therefore, $P(0)=$ $2 \beta_{0} p_{0}, M(0)=2 \beta_{0}$, and thus the right-hand side of Eq. (7) is $P_{v}=2 \beta_{0}\left(p_{0}+K\right)$.

Our simulations have the following goals:

(1) Confirm the momentum conservation Eq. (7).

(2) Determine whether the soliton oscillation frequency $\omega_{s}^{c c}$ of the CC theory is a good approximation for the soliton eigenfrequency $\omega_{s}$.

(3) Compare the $r$ dependence of $\omega_{s}^{c c}$, which is an analytical result from a linear stability analysis for stationary solutions [21], with the $r$ dependence of $\omega_{s}$.

(4) Search for a peak near $\omega_{-K}=K^{2}-\delta$ in the oscillation spectra.

(5) Check that this frequency belongs to an extended mode, in contrast to the intrinsic soliton oscillation mode.

(6) Confirm that the mode with $\omega_{-K}=K^{2}-\delta$ has zero velocity in the moving frame.

For $t>0$ we take $\psi(x, t)$ from the simulations and determine whether the conservation law (7) is fulfilled. Figure 1 shows $M(t), P(t)$, and $P_{v}(t)$, and we see that $P_{v}=P_{v}^{c c}=$ -0.32 with a numerical error of $\pm 4 \times 10^{-5}$. The same test was made for the other ICs, e.g. for $\beta_{0}=0.96$ and 1.52 .

In the second row of Fig. 1 we see that the soliton velocity $\dot{q}(t)$ and amplitude $\beta(t)$ exhibit oscillations. The discrete Fourier transform (DFT) of $\beta$ in the lowest row has a strong peak at $\omega_{s}=1.13$, very close to the prediction $\omega_{s}^{c c}=1.12$ of the CC theory, and there are several higher harmonics of $\omega_{s}$. Moreover, on the left shoulder of the main peak there is a very small peak at $\omega_{\mathrm{ph}}=1.0$ near the phonon band edge, plus the linear combination $\omega_{s}+\omega_{\mathrm{ph}}$.

In contrast to this, in the $P(t)$ spectrum (lowest row on the right), the phonon peak is much stronger than the soliton peak. This is easily explained by noticing that the soliton amplitude $\beta(t)$ is measured directly at the soliton, whereas $P(t)$ is obtained by an integration over the system.

Thus the simulation results clearly support that an oscillating soliton moves in the field of an extended phonon mode with a frequency close to the lower band edge.

In Fig. 2, we present simulations for very early times $0 \leqslant t \leqslant 15$ in order to study the transient behavior of the system. The upper left-hand panel shows that the soliton is deformed and starts to move directly after the start of the
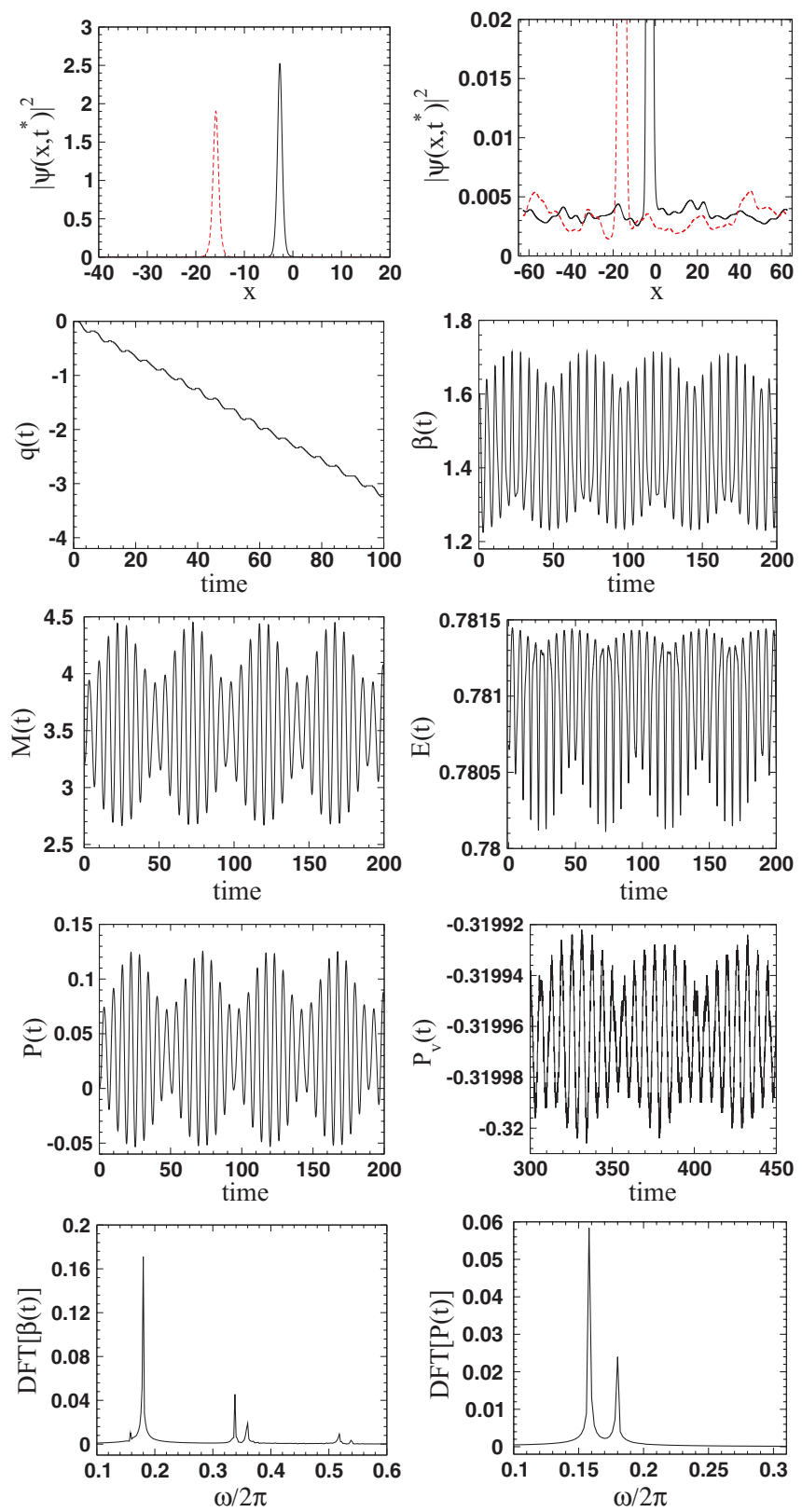

FIG. 1. (Colour online) Simulations for NLSE Eq. (1). First row panels (different scales): Soliton moving to the left at $t^{*}=83.3$ (black); $t^{*}=500$ (dashed-red). Second row: position and amplitude of the soliton. Third row: norm and energy. Fourth row: momentum in the laboratory frame and the quantity $P_{v}=P(t)+K M(t)$. Fifth row: Discrete Fourier transform (DFT). Parameters: $r=0.05, \delta=-1$, $K=-0.1, g=2$ with IC $\beta_{0}=1.6, p_{0}=q_{0}=\phi_{0}=0$. The energy of the soliton is constant with an error of $\pm 10^{-4}$. DFT of $\beta$ shows several frequencies, see text. DFT of $P$ shows two frequencies, 0.99 (height 0.058 ) and 1.13 (height 0.024 ).

simulation. The upper right-hand panel shows the beginning of the background oscillation due to the excitation of an extended phonon mode; see also the lower right-hand panel which presents the time evolution of the norm density at a point far away from the soliton. In the lower left-hand panel one can see how the momentum $P(t)$ is built up and starts to oscillate. 

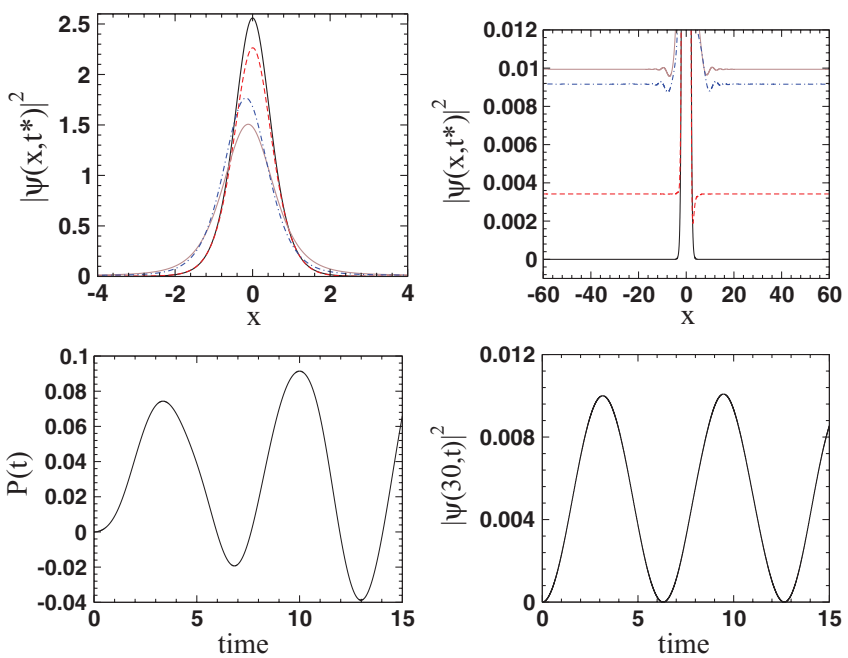

FIG. 2. (Color online) Simulations for short time for NLSE Eq. (1). First row panels (different scales): Soliton profiles at $t^{*}=0$ (black higher density), $t^{*}=1.25$ (dashed red line), $t^{*}=3$ (brown lower density), and $t^{*}=3.75$ (dot-dashed blue line). Second row: momentum and evolution of the density of the norm at $x=30$. Same parameters as in Fig. 1.

\section{A. Regimes of ICs for the simulations}

We choose $q_{0}=p_{0}=\phi_{0}=0$ and vary $\beta_{0}$, since this choice includes stationary solutions with $\beta_{0}=\beta_{s}$ ( $\beta_{s}$ will be given below).

We perform simulations for the following regimes:

(1) $\beta_{0}$ lies in one of the stability windows [17], but close to a boundary of the window, e.g., for the parameters in Fig. 1 there is a window for $\beta_{0} \geqslant 1.52$ [17]. In Fig. 1 we take $\beta_{0}=$ 1.6. Here the soliton oscillations are very anharmonic; see the DFT of $\beta(t)$ in Fig. 1. In contrast to this there are no higher harmonics in $P(t)$, which exhibits a strong peak near the phonon band edge $\omega_{0}=-\delta=1$.

(2) As in (a) but with $\beta_{0}$ not close to a boundary. Here the $\beta(t)$ oscillations are much more harmonic.

(3) Due to the goal number 3 above, we need simulations in the neighbourhood of stationary solutions. Here the soliton oscillations are small and practically harmonic [17]. The phonon peak turns out to be strong, both in $P(t)$ and $\beta(t)$. This helps with goal numbers 4 and 5 .

Stationary solutions of the CC equations were obtained in Ref. [17] by the ansatz $q(t)=q_{s}+v_{s} t, p(t)=p_{s}, \beta(t)=\beta_{s}$, and $\phi(t)=\phi_{s}-\alpha_{s} t$. By choosing a soliton at $q_{s}=0$ that is initially at rest $\left(p_{s}=0\right)$, a stable solution is found for $\phi_{s}=0$, and an unstable solution for $\phi_{s}=\pi$. Here $\beta_{s}$ is the solution of a transcendental equation, which is solved numerically. In the stable case, $\beta_{s}=1.732478$ for the parameter set $g=2, \delta=$ $-3, r=0.05, K=-0.1[17]$.

The stationary solution for the $\mathrm{CC}$ equations is naturally not an exact solution of the driven NLS Eq. (1), but it is very close. Our simulations yield very small oscillations in $q(t)$ and $\beta(t), M(t), P(t)$ around a mean trajectory and around mean values, respectively (see Fig. 3). The spectra of $\beta(t)$ and $P(t)$ show two peaks at $\omega_{s}=0.24$ and $\omega_{\text {ph }}=3.02$, close to the expected value $\omega_{K}=K^{2}-\delta=3.01$. In order to check the dependence on $K$, a simulation with $K=-0.4$ and $r=0.005$
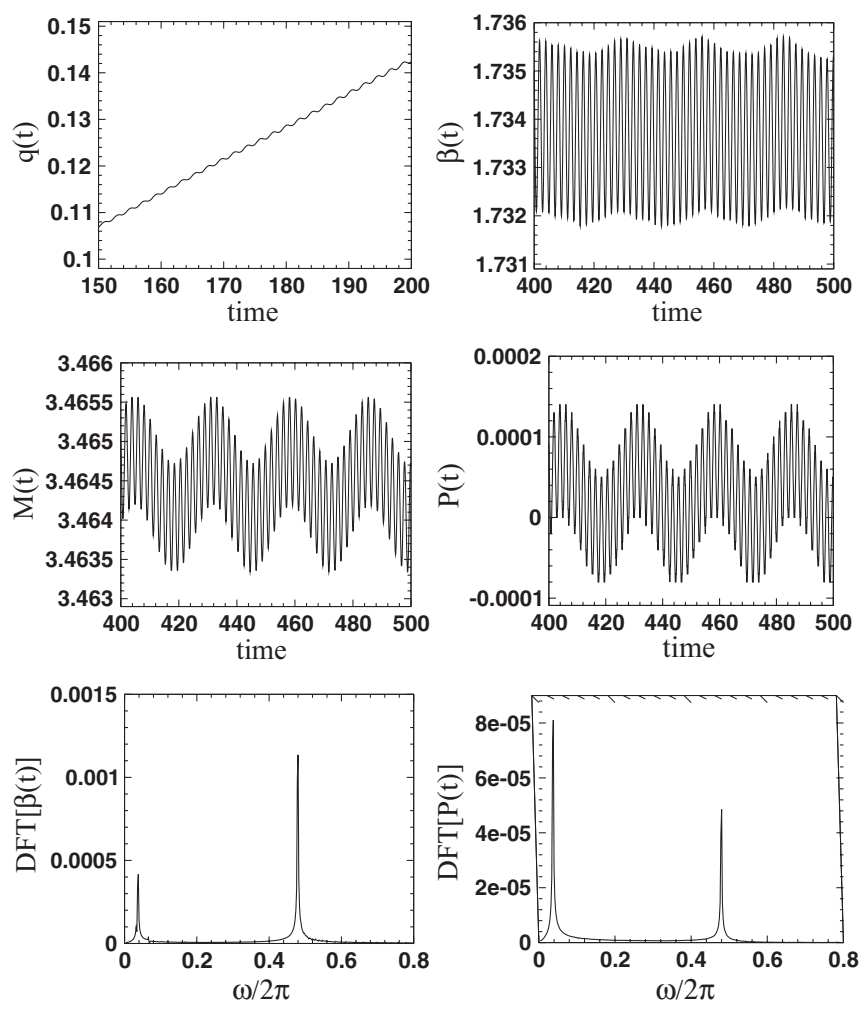

FIG. 3. NLSE Eq. (1). Upper panels: position and amplitude of the soliton. Middle panels: norm and momentum. Lower panels: DFT of $\beta$ shows oscillations with frequencies 3.02 (height 0.0011 ) and 0.23 (height $\left.1.6 \times 10^{-4}\right) ; P(t)$ shows oscillations with frequencies 0.24 (height $8 \times 10^{-5}$ ) and 3.02 (height $4.8 \times 10^{-5}$ ). $r=0.005, \delta=$ $-3, K=-0.1$, with IC $\beta_{0}=1.732094, p_{0}=q_{0}=\phi_{0}=0$.

is performed and yields $\omega_{s}=0.22$ and $\omega_{\mathrm{ph}}=3.17$, close to $\omega_{K}=3.16$.

In order to prove that $\omega_{\mathrm{ph}}$ is the frequency of an extended mode, we compute the DFT of the norm density $\left|\psi\left(x_{f}, t\right)\right|^{2}$ at fixed points $x_{f}$ far away from the soliton. These DFT all exhibit only one peak at $\omega_{\mathrm{ph}}=3.02$, close to the expected value $\omega_{K}=K^{2}-\delta=3.01$. An example is given in Fig. 4 .

Figure 4 shows results from a simulation for the autonomous NLSE Eq. (3). In the panel on the upper right-hand corner one can see that the soliton moves in the moving frame, but the phonon mode simply consists of an up and down oscillation of the background, as predicted.

In order to reveal the dependence of the soliton oscillation frequency $\omega_{s}$ on the driving strength $r$ and the other parameters, we compare with the result [21]

$$
\omega_{s}^{c c}=\left(K^{2}-\delta\right)^{1 / 4} \sqrt{2 \pi r} .
$$

This holds for small $r$ because it results from a linear stability analysis for stationary solutions of the CC equations [21]. Figure 5 shows very good agreement of $\omega_{s}$ with $\omega_{s}^{c c}$. For $r \geqslant 0.25$ the soliton is unstable.

Figure 5 also shows that $\omega_{\text {ph }}$ is always close to the phonon band edge $\omega_{0}=-\delta=3$. For $r=0.15,0.20, \omega_{\mathrm{ph}}$ is slightly smaller than $\omega_{0}$. This would mean that the amplitude of these excitations would be spatially attenuated. However, in our simulations we have not observed any attenuated phonons in front of the soliton or behind it. Taking into account the error 

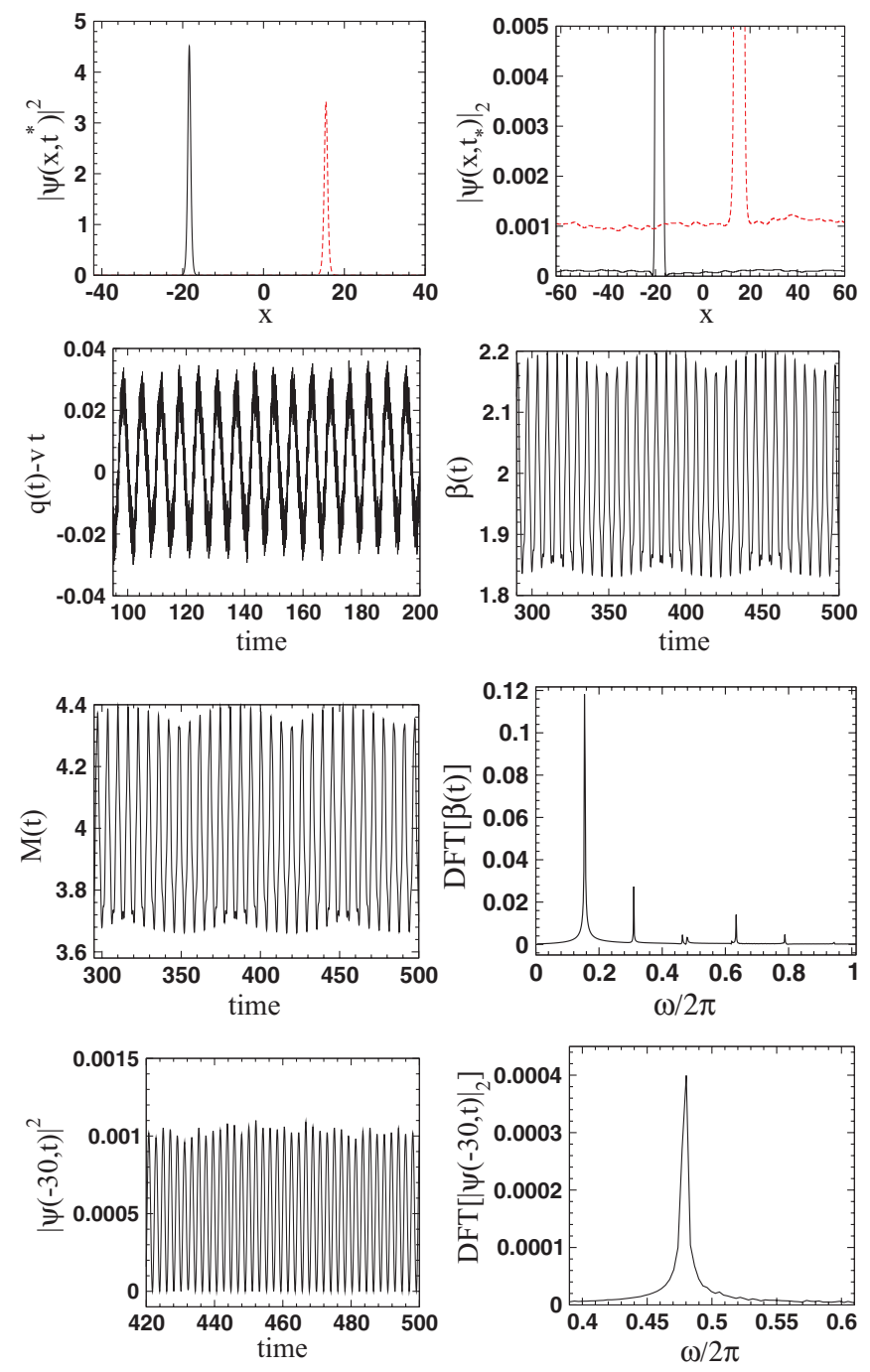

FIG. 4. (Color online) Autonomous NLSE Eq. (3). First row panels (different scales): Soliton moving to the left at $t^{*}=83.3$ (black); $t^{*}=500$ (dashed-red). Second row: $q(t)-\mathrm{v} t(\mathrm{v}=-0.22037$ denotes the mean velocity) and amplitude of the soliton. Third row: norm and DFT of the soliton's amplitude. DFT of $\beta$ shows frequencies 0.97 (height 0.118), 1.95 (height 0.027), and 3.98 (height 0.014). DFT of $M$ shows frequencies 0.97 (height 0.24 ), 1.95 (height 0.055 ), and 3.98 (height 0.028 ). Fourth row: Oscillations of the density of the norm at a fixed point show the frequency 3.02 (height 0.00039 ). The momentum is conserved, $P_{v}=P_{v}^{c c}=-0.42$ with a numerical error of $10^{-4}$. Parameters: $r=0.05, \delta=-3, K=-0.1, \theta=0$, with IC $\beta_{0}=2.16, p_{0}=q_{0}=\phi_{0}=0$.

bars of 0.02 for $\omega_{\mathrm{ph}}$, we therefore conclude that the excitations for $r=0.15,0.2$ could also belong to the phonon band.

Finally, we want to compare our spectrum for a moving soliton (Fig. 5) with the results from the numerical linear stability analysis for the stable static solution $u_{-}(y, t)$ of the autonomous Eq. (3) [11]. The factor $K^{2}-\delta=c^{2}$ can be eliminated by scaling time and space by $t=\tau_{1} / c^{2}, y=\xi / c$, and $u=c \phi$. This yields the equation that was investigated in Ref. [11],

$$
i \phi_{\tau_{1}}+\phi_{\xi \xi}+2|\phi|^{2} \phi-\phi=h,
$$

where $h=r / c^{3}$.

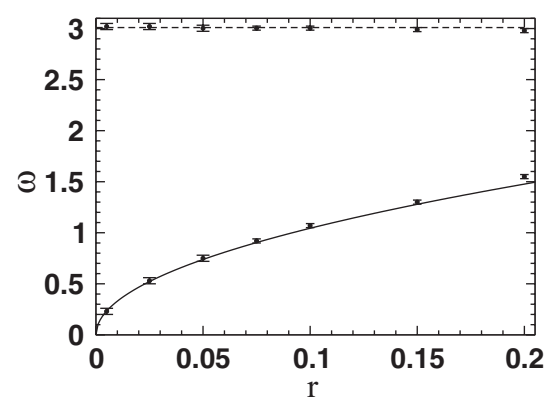

FIG. 5. Frequencies of the soliton and phonon oscillations as a function of the driving strength $r$. The solid line represents the curve $\omega=\sqrt{2 \pi r}\left(K^{2}-\delta\right)^{1 / 4}$ versus $r$, which results from a linear stability analysis for stationary solutions of the CC equations [21]. The points with their error bars stem from the DFT of both $\beta(t)$ and $P(t)$ in the simulations. The dashed line represents the phonon band edge at $\omega_{0}=$ $-\delta=3$. Other parameters: $g=2, K=-0.1, \beta_{0}=1.732478, p_{0}=$ $q_{0}=\phi_{0}=0$. For $r \geqslant 0.25$ the soliton is unstable.

Unfortunately, Fig. 1 of Ref. [11] is only schematic, and hence a quantitative comparison cannot be made. However, a qualitative comparison yields the following:

(1) For $0<h<h_{c}=0.07749$ there are two eigenfrequencies in the gap between the phonon continuum and the ground state, and for $h>h_{c}$ the soliton is unstable.

(2) For small $h$, the lower frequency is proportional to $\sqrt{h}$, the same behavior as in our Fig. 5 .

(3) The higher frequency mode is detached from the continuum and decreases linearly with $h$, for small $h$. In contrast to this, our phonon mode is always close to the edge of the continuum.

(4) The two modes meet at $h=h_{c}$. This cannot occur in Fig. 5, because here the soliton already becomes unstable in the simulations for $r \geqslant 0.25$. This value is much smaller than the critical value $r_{c}=c^{3} h_{c}=0.4047$, which corresponds to $h_{c}=0.07749$.

Finally, we investigate what happens when the external force $f(x)=r \exp (-i K x)$ is switched on adiabatically. We multiply $f(x)$ by the switching function $h(t)=1-(1+$ $t / \tau) \exp (-t / \tau)$ with the properties $h(0)=\dot{h}(0)=0$, and $h \rightarrow$ 1 for $t \gg \tau$. We choose the same parameters as in Fig. 1 and assign $\tau=100$. Figure 6 shows that after a transient time of about 500, $\beta(t)$ and $P(t)$ exhibit practically the same intrinsic frequency $\omega_{s}=0.23$ as in Fig. 1. Moreover, the DFT do not contain a second frequency at 3.02 near the edge of the phonon band, in fact there is only one peak. This is confirmed by the norm density in the middle right-hand panel, which shows no background oscillations. This means that only the intrinsic soliton mode $\omega_{s}$ is excited when the driving force is switched on adiabatically (i.e., sufficiently slowly). The amplitude $a_{\beta}$ of the soliton oscillations is very small $\left(2 \times 10^{-3}\right)$ and can be further reduced by fine tuning the IC $\beta_{0}$. For example, for $\beta_{0}=1.731878$, the oscillations nearly vanish $\left(a_{\beta}=2 \times 10^{-4}\right)$ and the soliton velocity is nearly constant $(v=0.0078)$. This means that we practically have a traveling soliton without oscillations, i.e., a stationary solution. Such solutions have recently been obtained numerically by path-following them in the parameter space [27]. 

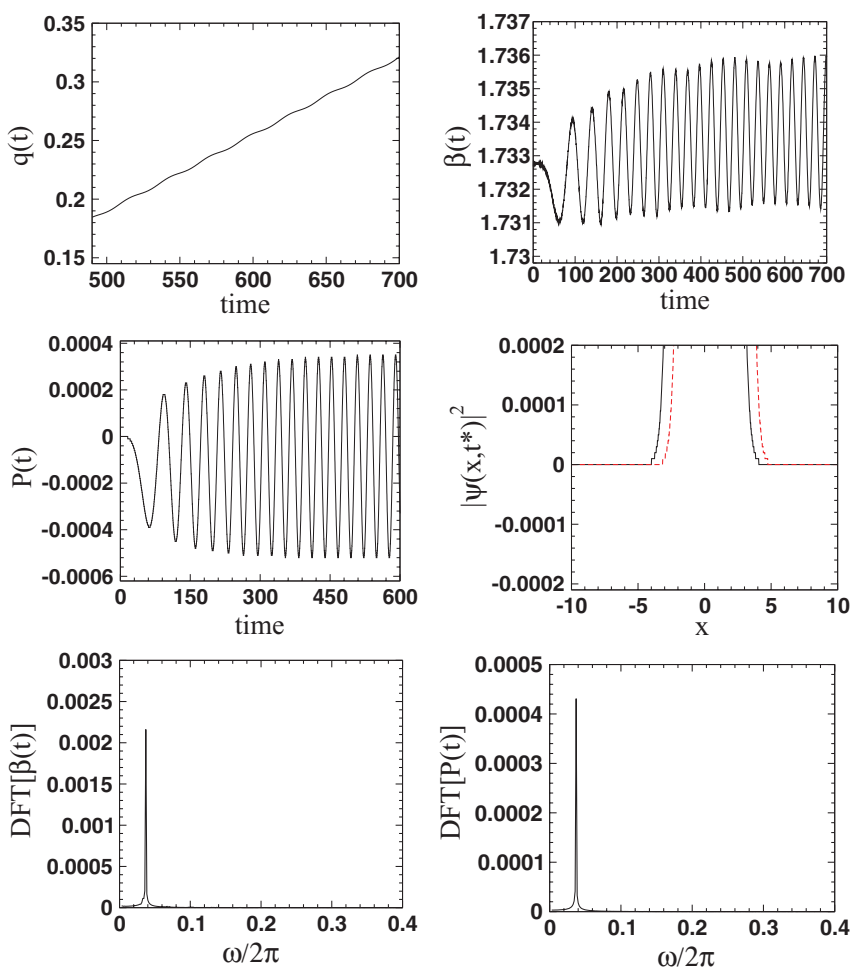

FIG. 6. (Color online) Simulations for NLSE Eq. (1) where the force is adiabatically switched on by $h(t) r \exp (-i K x)$ with $h(t)=1-(1+t / \tau) \exp (-t / \tau)$ with $\tau=100$. First row panels: $q(t)$ and $\beta(t)$. Second row: $P(t)$ and norm density for $t^{*}=233.3$ (black); 1400 (dashed-red). Third row: DFT of $\beta$ and $P$ show the frequency 0.23 [height 0.0022 in $\beta(t)$ and 0.00043 in $P(t)$ ]. Parameters: $r=0.005, \delta=-3, K=-0.1, g=2$ with IC $\beta_{0}=1.732478, p_{0}=$ $q_{0}=\phi_{0}=0$.

\section{CONCLUSIONS}

Our simulations for the cubic NLSE Eq. (1) with the external force $f(x)=r \exp (-i K x)$ exhibit traveling solitons which oscillate, i.e., the soliton's amplitude $\beta(t)$ and velocity $\dot{q}(t)$ oscillate around mean values of $\beta$ and $\dot{q}$; moreover, the norm $M(t)$ and total momentum $P(t)$ of the system also oscillate around mean values. The spectra of the oscillations generally exhibit two strong peaks: (1) The frequency $\omega_{s}$ is close to $\omega_{s}^{c c}$, which is the frequency of an intrinsic soliton mode predicted by a collective coordinate theory [17]. This theory generally yields anharmonic oscillations. This is confirmed by the simulations, for example, the spectrum of $\beta(t)$ in Fig. 1 exhibits the higher harmonics $2 \omega_{s}$ and $3 \omega_{s}$. However, $P(t)$ does not contain higher harmonics. (2) Generally, a second strong peak is observed at $\omega_{\mathrm{ph}}$, which is close to the lower band edge $\omega_{0}=-\delta$ of the continuum of linear excitations (phonons). This phonon mode is excited for the following reason: Initially the soliton is at rest. Due to the driving force the soliton is accelerated and deformed and starts to oscillate. When the soliton starts to move, it acquires a momentum and the above phonon mode is excited such that the total momentum in a frame moving with the velocity $v_{f}=-2 \mathrm{~K}$ is conserved (this conservation law has been proven in Ref. [21]). We have shown that, in this moving frame, the phonon mode with $\omega_{K}=K^{2}-\delta \approx \omega_{\mathrm{ph}}$ does not move, thus the total momentum is equal to the soliton momentum. The above phonon mode is an extended mode which represents a homogeneous up and down oscillation of the background (Fig. 4, first row on the right). Moreover, we have computed the norm density at fixed points far away from the soliton and we see in the spectrum only one peak near $\omega_{K}=K^{2}-\delta$, as expected (Fig. 4, fourth row on the right).

Summarizing, we conclude that the intrinsic soliton mode and the above specific phonon mode together fully explain the observed oscillations.

\section{ACKNOWLEDGMENTS}

F.G.M. is grateful for the hospitality of the Mathematical Institute of the University of Seville (IMUS) and of the Theoretical Division and Center for Nonlinear Studies at Los Alamos National Laboratory, and for financial support from the Plan Propio of the University of Seville, from Junta de Andalucia IAC11-III-11965, and from the MICINN (Spain) through FIS2011-24540. N.R.Q. acknowledges financial support from the Humboldt Foundation through a Research Fellowship for Experienced Researchers (SPA 1146358 STP), and from the MICINN through FIS2011-24540, and by Junta de Andalucia under Projects No. FQM207, No. P11-FQM7276, and No. P09-FQM-4643.

\section{APPENDIX: PHONONS IN THE MOVING FRAME}

We first transform the solution in the laboratory frame to the moving frame, considering

$$
\psi(x, t)=c e^{i\left(k x-\omega_{k} t\right)}-\frac{r}{\omega_{K}} e^{-i K x},
$$

with $\omega_{k}=k^{2}-\delta$ and $\omega_{K}=K^{2}-\delta$. This equation can be rewritten as

$$
\psi(x, t)=e^{-i K x} u(x, t), \quad u(x, t)=c e^{i\left([k+K] x-\omega_{k} t\right)}-\frac{r}{\omega_{K}} .
$$

Using $x=y-2 K t, u(y, t)$ becomes a phonon mode in the moving frame,

$$
u(y, t)=c e^{i\left(\tilde{k} y-\tilde{\omega}_{\tilde{k}} t\right)}-\frac{r}{\omega_{K}},
$$

with $\tilde{k}=k+K$ and $\tilde{\omega}_{\tilde{k}}=\omega_{k}+2 K \tilde{k}=-\delta+\tilde{k}^{2}+K^{2}$. Indeed, by inserting (A3) into the linearized autonomous NLSE, we obtain $\tilde{\omega}_{\tilde{k}}$ as above.

Now we calculate the phonon momentum in the moving frame by inserting (A3) into (8) and integrating between $-L$ and $L$ :

$$
P_{v}^{\mathrm{ph}}=2 \tilde{k}|c|^{2} L-\frac{2 r}{\omega_{K}}[a \cos (\tilde{\omega} t)+b \sin (\tilde{\omega} t)] \sin (\tilde{k} L) .
$$

Clearly, $P_{v}^{\text {ph }}$ is independent of $t$ only if $\sin (\tilde{k} L)=0$, i.e., $\tilde{k}_{n}=$ $n \pi / L$, with $n=0, \pm 1, \pm 2, \ldots$. Notice that for these $\tilde{k}_{n}$, the periodic BC $u(L, t)=u(-L, t)$ are fulfilled. Moreover, $P_{v}^{\mathrm{ph}}=$ 0 only if $\tilde{k}=0$; i.e. $k=-K$ and $\tilde{\omega}_{0}=-\delta+K^{2}=\omega_{K}$, which is the same frequency in both frames. 
[1] Y. Kivshar and B. Malomed, Rev. Mod. Phys. 61, 763 (1989).

[2] B. A. Malomed, in Progress in Optics, edited by E. Wolf, Vol. 43 (North-Holland, Amsterdam, 2002), p. 71.

[3] L. D. Faddeev and L. Takhtajan, Hamiltonian Methods in the Theory of Solitons, Classics in Mathematics (Springer-Verlag, Berlin, 2007).

[4] I. V. Barashenkov, M. M. Bogdan, and V. I. Korobov, Europhys. Lett. 15, 113 (1991).

[5] M. Bondila, I. V. Barashenkov, and M. M. Bogdan, Physica D 87, 314 (1995)

[6] R. Scharf and A. R. Bishop, Phys. Rev. E 47, 1375 (1993).

[7] I. V. Barashenkov, E. V. Zemlyanaya, and M. Bär, Phys. Rev. E 64, 016603 (2001).

[8] D. Poletti, E. A. Ostrovskaya, T. J. Alexander, B. Li, and Y. S. Kivshar, Physica D 238, 1338 (2009).

[9] D. J. Kaup and A. C. Newell, Proc. R. Soc. London A 361, 413 (1978); Phys. Rev. B 18, 5162 (1978).

[10] I. V. Barashenkov and E. V. Zemlyanaya, Physica D 132, 363 (1999).

[11] I. V. Barashenkov, T. Zhanlav, and M. M. Bogdan, in Nonlinear World, IV International Workshop on Nonlinear and Turbulent Processes in Physics, edited by V. G. Bar'yakhtar, M. Chernousenko, N. S. Erokhin, A. G. Sitenko, and V. E. Zakharov (World Scientific, Singapore, 1990), pp. 3-9.

[12] G. Cohen, Phys. Rev. E 61, 874 (2000).

[13] V. M. Vyas, T. S. Raju, C. N. Kumar, and P. K. Panigrahi, J. Phys. A 39, 9151 (2006).
[14] P. S. Lomdahl and M. R. Samuelsen, Phys. Rev. A 34, 664 (1986).

[15] B. A. Malomed, Phys. Rev. E 51, R864 (1995).

[16] K. Nozaki and N. Bekki, Physica D 21, 381 (1986).

[17] F. G. Mertens, N. R. Quintero, and A. R. Bishop, Phys. Rev. E 81, 016608 (2010).

[18] F. G. Mertens, N. R. Quintero, I. V. Barashenkov, and A. R. Bishop, Phys. Rev. E 84, 026614 (2011).

[19] U. Peschel, O. Egorov, and F. Lederer, Opt. Lett. 15, 1909 (2004).

[20] A. Gorbach, S. Denisov, and S. Flach, Opt. Lett. 31, 1702 (2006).

[21] F. Cooper, A. Khare, N. R. Quintero, F. G. Mertens, and A. Saxena, Phys. Rev. E 85, 046607 (2012).

[22] L. A. Lugiato and R. Lefever, Phys. Rev. Lett. 58, 2209 (1987); W. J. Firth, G. K. Harkness, A. Lord, J. M. M. McSloy, D. Gomila, and P. Colet, J. Opt. Soc. Am. B 19, 747 (2002).

[23] M. Haeltermann, S. Trillo, and S. Wabnitz, Opt. Lett. 17, 745 (1992); Opt. Commun. 91, 401 (1992).

[24] S. Wabnitz, Opt. Lett. 18, 601 (1993); J. Opt. Soc. Am. B 13, 2739 (1996).

[25] G. M. Reznik and V. Zeitlin, Phys. Rev. Lett. 99, 064501 (2007); Nonlin. Processes Geophys. 16, 381 (2009).

[26] I. V. Barashenkov, and Yu. S. Smirnov, Phys. Rev. E 54, 5707 (1996).

[27] I. V. Barashenkov and E. V. Zemlyanaya, J. Phys. A: Math. Theor. 44, 465211 (2011).

[28] A. Kovalev, F. G. Mertens, and H. J. Schnitzer, Eur. Phys. J. B 33, 133 (2003). 